\title{
Efecto de la instrucción explícita sobre la interpretación de implicaturas en aprendientes angloparlantes de español como segunda lengua
}

\author{
Gloria Macarena Toledo Vega \\ Profesora Facultad de Letras \\ Pontificia Universidad Católica de Chile \\ Vicuña Mackenna \\ 4860, Macul, Santiago \\ E-mail: gtoledo@uc.cl
}

\author{
Fernando Antonio Soto Celis \\ Jefe de Programa Transversal de Lenguaje e \\ Inglés \\ Instituto DUOC UC \\ Calle Nueva \\ 1660, Huechuraba, Santiago \\ E-mail: fsotoc@duoc.cl
}

\author{
EFECTO DE LA INSTRUCCIÓN EX- \\ PLÍCITA SOBRE LA INTERPRETA- \\ CIÓN DE IMPLICATURAS EN \\ APRENDIENTES ANGLOPARLAN- \\ TES DE ESPAÑOL COMO SE- \\ GUNDA LENGUA
}

RESUMEN: Este estudio busca determinar el efecto de la instrucción explícita sobre la comprensión de implicaturas en español como segunda lengua (ESL); determinar si existe una relación entre la competencia gramatical de los aprendientes y su comprensión de implicaturas; y observar si existe un tipo de implicatura que muestre mayor dificultad en la adquisición. En la muestra participaron 23 aprendientes de ESL que fueron divididos en seis grupos (tres experimentales y tres de control) de acuerdo a su nivel de competencia en español. Los tres grupos experimentales participaron de una sesión de 30 minutos de instrucción explícita sobre implicaturas. Los resultados no muestran respaldo estadístico, sin embargo, se observa una leve mejora entre los participantes de mayor nivel de competencia gramatical, lo cual va en línea con otros estudios respecto a que quienes más se benefician de la instrucción para la comprensión de implicaturas, son los aprendientes de niveles más avanzados.

PALABRAS CLAVES: instrucción explícita, implicaturas, español como segunda lengua, competencia gramatical, pragmática.

SUMARIO: 1. Introducción. 2. Lineamientos teóricos o. 3. Marco metodológico. 4. Resultados. 5. Discusión de resultados y conclusiones.
EFFECT OF EXPLICIT INSTRUCTION ON IMPLICATURE'S INTERPRETATION IN ENGLISHSPEAKING LEARNERS OF SPANISH AS A SECOND LANGUAGE

ABSTRACT: This paper aims to determine first the effect of explicit instruction on the comprehension of implicatures in Spanish as a Second Language (SSL); second, whether there is a connection between learners' grammatical accuracy and their ability to understand such implicatures; and third, if there is a type of implicature which proves more difficult/problematic to acquire. The sample was formed by 23 participants, learners of SSL. They were divided into 6 groups (3 experimental groups and 3 control groups) according to their competence level in Spanish. The 3 experimental groups participated in a 30- minute-long explicit instruction session about implicatures. Even though the statistics did not support the results, there was a slight improvement of the group with the highest competence level. This result is line with previous studies showing that highest level learners profit more from explicit instruction to interpret implicatures.

KEY WORDS: explicit instruction, implicatures, Spanish as Second Language, grammatical competence, Pragmatics.

SUMMARY: 1 . Introduction. 2 . Theoretical guidelines o. 3. Methodological framework. 4. Results. 5. Discussion of results and conclusions.

EFFET D'UNE INSTRUCTION
EXPLICITE
L'INTERPRETATION
IMPLICATURES CHER
APPRENANTS ANGLOPHONES EN
ESPAGNOL COMME LANGUE
SECONDE

RÉSUMÉ: Cette étude cherche à déterminer l'effet d'une instruction explicite sur la compréhension des implicatures en espagnol comme langue seconde (ELS); à déterminer s'il existe une relation entre la compétence grammaticale des apprenants et leur compréhension des implicatures; et à observer s'il existe un type d'implicature qui présente une plus grande difficulté dans l'acquisition. L'échantillon a impliqué 23 apprenants d'ELS qui ont été divisés en six groupes (trois expérimentaux et trois témoins) selon leur niveau de compétence en espagnol. Les trois groupes expérimentaux ont participé à une session de 30 minutes d'enseignement explicite sur les implicatures. Les résultats ne montrent pas de soutien statistique mais il y a une légère amélioration chez les participants de plus haut niveau de compétence grammaticale, ce qui est conforme à d'autres études qui montrent que ceux qui bénéficient le plus des l'instructions pour la compréhension des implicatures sont les apprenants des niveaux les plus avancés.

MOTS CLÉS: instruction explicite, implicatures, L'espagnol comme langue seconde, compétence grammaticale, Pragmatique.

SOMMAIRE: 1 . Introduction. 2 . Lignes directrices théoriques o. 3 Cadre méthodologique. 4. Résultats 5. Discussion des résultats et des conclusions. $\begin{array}{ll}\text { Fecha de Recepción } & 05 / 09 / 2017 \\ \text { Fecha de Revisión } & 16 / 07 / 2018 \\ \text { Fecha de Aceptación } & 31 / 07 / 2018 \\ \text { Fecha de Publicación } & 01 / 12 / 2018\end{array}$ 


\title{
Efecto de la instrucción explícita sobre la interpretación de implicaturas en aprendientes angloparlantes de español como segunda lengua
}

\author{
Gloria Macarena Toledo Vega \& Fernando Antonio Soto Celis
}

\section{INTRODUCCIÓN}

La inclusión del componente pragmático en la enseñanza - aprendizaje de una segunda lengua ha sido un tema cuyo desarrollo data de unos 20 años, pero que aun plantea algunas dificultades. Rose (1994) sugiere que la mayoría de los aprendientes de una lengua extranjera no usan la competencia pragmática y los que sí la usan lo hacen con otros hablantes no nativos. Por otra parte, es una tarea dificil determinar cuál sistema pragmático sirve de modelo para enseñar en una clase de lengua extranjera ${ }^{1}$. De hecho, muchas dificultades guardan relación con los usos (o no-usos) que se les darán a los conocimientos pragmáticos en la lengua meta pues no siempre que un aprendiente adquiere una lengua extranjera terminará usándola necesariamente. Un último tipo de inconveniente tiene que ver con que los docentes de una segunda lengua no son usualmente hablantes nativos. Si bien algunos autores como Widdowson (1992) o Medgyes (1992) describen posibles beneficios sobre el desarrollo de las competencias gramaticales y léxicas cuando los aprendientes trabajan con un profesor no nativo, Rose (1994) asegura que, en lo que respecta al desarrollo pragmático, trabajar con un profesor que es hablante nativo de la lengua que se estudia tiene mayores ventajas.

En vista de las dificultades recién señaladas este estudio tiene como objetivo determinar y describir el efecto que tiene la instrucción explícita de implicaturas (Grice 1975) en la interpretación que hacen de ellas los aprendientes de español como segunda lengua (ESL) ${ }^{2}$. También busca saber si existe una relación entre la competencia gramatical y la comprensión de implicaturas y si existe algún tipo de implicatura más difícil de inferir que otro. La instrucción explícita será entendida como un conjunto de actividades expositivas que explican y ejemplifican el concepto de implicatura y que contrasta con otros tipos de actividades en las cuales los aprendientes son expuestos a situaciones en las que se espera que interpreten implicaturas a través de la inducción.

\footnotetext{
${ }^{1}$ Nos referimos al concepto de español como lengua extranjera en oposición al de español como segunda lengua, entendiendo que este último se aprenderá o adquirirá en un contexto de inmersión específico, lo cual facilitará la tarea de determinar el sistema sociopragmático al que adscribirá el profesor de ESL, ya que lo más probable es que sea el de la comunidad de inmersión.

${ }^{2}$ Usamos el concepto de segunda lengua (SL) porque este estudio se llevó a cabo en un contexto de inmersión con aprendientes de ESL del Programa de Español de la Pontificia Universidad Católica de Chile.
} 
La metodología de este estudio descriptivo y correlacional se basó en un pre test, una clase de instrucción explícita sobre implicaturas y un post test para medir los efectos de la clase. Para validar los tests los aplicamos antes a hablantes chilenos que sirvieron como grupo de control. Para comprobar el efecto sobre la comprensión de implicaturas el porcentaje de acierto en el pretest se comparó con el porcentaje del postest y se aplicaron pruebas estadísticas para establecer si existían diferencias significativas en los resultados entre e intra grupos. Posteriormente se compararon los porcentajes de aciertos según el tipo de implicatura inferido por todos los participantes en ambas evaluaciones, para determinar si existía algún tipo de implicatura cuya comprensión fuera más fácil o dificil de lograr.

\section{LINEAMIENTOS TEÓRICOS}

\subsection{PAPEl de LA ENSEÑANZA EN El DESARROLlo DE LA CONSCIENCIA PRAGMÁtica}

Basándose en la Noticing Hypothesis de Schmidt (1990), Bardovi-Harlig (1996) afirma que darse cuenta de las formas lingüísticas (noticing) es el primer nivel de conciencia, independiente de un segundo nivel de comprensión (understanding) en el que los aprendientes reconocen un principio general, regla o patrón. Esta investigación se concentra en la conciencia en el primer sentido (noticing). Se entenderá conciencia pragmática como la información que el aprendiente ha obtenido percatándose del uso del lenguaje y lo apropiado de los enunciados en contextos específicos.

Entre quienes han investigado de forma más extensiva la comprensión de implicaturas está Bouton (1994), quien comparó el grado en el que 436 estudiantes internacionales hablantes no nativos del inglés, interpretaban implicaturas en inglés americano en relación con hablantes nativos que también participaron en su estudio. De los resultados obtenidos en su trabajo se desprende que los hablantes no nativos pueden desarrollar un alto nivel de desempeño al interpretar implicaturas en inglés si disponen del tiempo suficiente. Para resolver la duda respecto a la cantidad de tiempo suficiente, Bouton llevó a cabo un segundo estudio en el que seleccionó 304 estudiantes que fueron agrupados según el tiempo que llevaban estudiando en la Universidad de Illinois. Un grupo quedó conformado por estudiantes que llevaban 17 meses de clases, un segundo grupo comprendía estudiantes que llevaban 33 meses y finalmente un tercer grupo estuvo compuesto por estudiantes que habian llegado hace cuatro años. Si bien Bouton encontró diferencias en el desempeño de los distintos grupos, que indicaban un progreso directamente proporcional al tiempo de exposición a la lengua objeto, dichas diferencias no eran estadísticamente significativas, con lo cual no podía afirmarse que los resultados no se debieran al azar.

Obviando las estadísticas, Bouton examinó en detalle qué ocurría con cada tipo de implicatura para determinar cuál causaba mayor dificultad al ser interpretada por los aprendientes. Las implicaturas que incluyó en su estudio fueron divididas en dos tipos: las que son más convencionales o 
dependientes de fórmulas y las que no. Las implicaturas que se producen al transgredir la máxima de relevancia tienden a ser menos convencionales, es decir, su interpretación depende de la relación idiosincrásica entre un enunciado particular y su contexto específico, lo que genera mayor dificultad en la interpretación ya que los hablantes no nativos deben aprender cuáles son los aspectos culturales relevantes en los que está basada la implicatura. Como contraste, las implicaturas que se producen por la transgresión de otras máximas ${ }^{3}$, dependen de algún tipo de fórmula estructural, semántica o pragmática.

Siguiendo de cerca la investigación de Bouton, Kubota (1995) demuestra que los grupos experimentales que recibieron instrucción en su estudio generaron mejores respuestas que el grupo de control. Con estos resultados Kubota afirma que enseñar a inferir implicaturas conversacionales, ya sea a través de explicaciones explícitas o mediante tareas de concienciación, facilita el desarrollo de la conciencia pragmática. Otros autores cuyas investigaciones apoyan la instrucción explícita son McManus (2017); Cox (2015); Cignetti y Di Giuseppe (2015); y Fordyce (2013). Cignetti y Di Giuseppe (2015) aseguran que los principios que regulan la comprensión de implicaturas funcionan de distintas maneras en distintas sociedades, grupos sociales y situaciones, obstaculizando la comunicación intercultural y creando la necesidad de intervenir pedagógicamente. La comparación que ellas hacen entre los estudios de Bouton (1994) y Kubota (1995) permite resaltar la importancia de tres factores que influyen en la comprensión de implicaturas por parte de los aprendientes: extensión en el tiempo del tratamiento, nivel de competencia en la lengua objeto y ambiente de aprendizaje.

Martínez-Flor y Alcón (2004) sugieren beneficios significativos, tanto de la instrucción explícita como la implícita, en el desarrollo de la conciencia pragmática de los aprendientes. En una línea similar, Eslami-Rasekh (2004) afirma que las actividades que pueden conducir al desarrollo pragmático de los aprendientes son clasificables en actividades para aumentar la conciencia pragmática y actividades que ofrecen oportunidades para la práctica comunicativa. Por último, Clennell (1999), propone ver cómo los significados sociopragmáticos están contenidos en el discurso y cómo la comunicación efectiva depende de la interacción y de la negociación mutua, lo cual apoya la implementación de actividades comunicativas, como sugiere Eslami-Rasek (2004).

\subsection{INTERFAZ GRAMÁTICA-PRAGMÁTICA}

En el presente estudio gramática y pragmática se entienden como lo hace Bardovi-Harlig (1996); la gramática se refiere a la precisión (accuracy) de la estructura, incluyendo la morfología y la sintaxis, mientras la pragmática se enfoca en el uso del lenguaje y se preocupa de lo apropiado de los enunciados dadas situaciones, hablantes y contextos específicos.

\footnotetext{
${ }^{3}$ Máximas de cantidad, calidad y relación (Grice, 1975).
} 
Los estudios respecto a la interfaz gramática y pragmática van por dos lineas opuestas: aquellos que sostienen que las funciones pragmáticas se adquieren antes que las formas gramaticales en L2 y los que sostienen que el conocimiento gramatical precede al saber pragmático. La investigación de Cook y Liddicoat (2002) comparó niveles de lengua altos y bajos para determinar el desarrollo de la consciencia pragmática, atendiendo a peticiones de angloparlantes australianos. Sus investigaciones sugieren que un mayor nivel de lengua se traduce en una mayor habilidad para interpretar correctamente los enunciados de la petición. La hipótesis de que la gramática precede a la pragmática se sostiene en tres resultados observados en distintos estudios: (a) los aprendientes demuestran un conocimiento de una estructura gramatical determinada, pero no lo usan para expresar o modificar la fuerza ilocutiva; (b) los aprendientes demuestran tener conocimiento de las estructuras gramaticales y las usan para expresar funciones pragmalingüísticas, pero que no están convencionalizadas en la lengua meta y (c) los aprendientes demuestran conocimiento de las estructuras gramaticales y de las funciones pragmáticas, pero de todas formas se dirigen desde la forma a la función hacia un uso sociopragmático que no es el de la L2 (Kasper y Rose, 2002: p. 175).

Una confrontación de los estudios de Bardovi - Harlig y Dörnyei (1998) y el de Niezgoda y Röver (2001) sobre la conciencia gramatical y pragmática en contextos SL y LE, sugiere que la conciencia pragmática y gramatical son completamente independientes. En el estudio de 1998, los estudiantes más avanzados e inmersos en un contexto natural de adquisición, mostraban más conciencia respecto a los errores pragmáticos y atribuían a éstos mayor importancia que a los fallos gramaticales. El trabajo de Niezgoda y Röver expuso resultados divergentes, ya que los aprendientes con menor nivel de lengua fueron quienes detectaron más errores pragmáticos y les dieron más peso. Por otra parte, el estudio de Koike (1989) muestra que ya que la competencia gramatical no se puede desarrollar tan rápidamente como requieren los conceptos pragmáticos, estos se expresan de acuerdo al nivel de complejidad gramatical adquirido.

Buena parte de los estudios que apoyan la idea de que la pragmática precede a la gramática se basan en el modelo bidimensional de Bialystok (1993) que propone la presencia de un sistema pragmático preexistente (de la lengua materna). La investigación sobre pragmática e interlengua, así como de otros campos de la adquisición del lenguaje, tiende a mostrar la primacía de la pragmática sobre la competencia gramatical en aprendices adultos. Esta concepción se apoya en las observaciones de que los aprendices adquieren conceptos de pragmática de la L2, pero su competencia gramatical se desarrolla más lentamente (Pearson, 2006).

La relación entre el desarrollo pragmático y el gramatical, en el contexto de adquisición de segundas lenguas o lenguas extranjeras, reside en lo que advierte Schmidt (1993a, 1993b) respecto a que es necesaria la atención a las formas lingüísticas, los significados funcionales y los rasgos contextuales relevantes para que ocurra la adquisición pragmática, pues aquello que los aprendientes notan es lo que se transforma en intake para la adquisición. 
En este sentido creemos que, si hacemos conscientes a los aprendientes sobre el uso pragmático de ciertos ítems gramaticales, estos últimos podrán incorporarse al saber pragmático en una L2. Como sostiene Gallardo-Paúls (2005), es difícil desvincular la relación que existe entre la organización interna de la pragmática y la gramática, en efecto, ciertas inferencias participan de una naturaleza dual que a la vez es sintáctica y pragmática (2005: S67).

\section{PRopuesta de Clasificación}

\subsection{TIPO DE ESTUDIO}

Esta investigación tiene un alcance descriptivo, ya que busca determinar el efecto de la instrucción explícita, es decir, la explicación directa del concepto de implicatura para la comprensión de estas en el aprendizaje de ELE. Por otro lado, tiene un alcance correlacional dado que intenta cuantificar la relación entre la competencia gramatical observada en los aprendientes y su comprensión de implicaturas, y la relación entre distintos tipos de implicatura y la dificultad para comprenderlas. Se trata de un estudio cuantitativo que utilizó un diseño experimental dada la manipulación intencional de una variable independiente, que corresponde a la instrucción explícita y a la medición de una variable dependiente relacionada con la comprensión de implicaturas en grupos experimentales y un grupo de control.

\subsection{OBJETIVOS}

Lo que esta investigación busca determinar es: (i) qué efecto tiene la instrucción explícita sobre la comprensión de implicaturas conversacionales en español por parte de aprendientes de ELE; (ii) si existe una relación entre la competencia gramatical de los aprendientes y su comprensión de implicaturas conversacionales en español y (iii) si existe un tipo de implicatura que cause mayor dificultad en la comprensión.

\subsection{PARTICIPANTES}

En su totalidad, la muestra se constituyó con 23 participantes. Todos ellos hablaban inglés (18 como lengua materna) y en promedio habían estado estudiando español durante 3,4 años.

Para analizar la correlación entre comprensión de implicaturas y nivel de lengua se constituyó un total de seis grupos de participantes divididos en tres niveles de lengua (B1, B2 y C1).

En cada nivel hubo un grupo experimental que recibió tratamiento de instrucción explícita y uno de control que no lo recibió, lo cual permitió comparar el desempeño de los aprendientes en cada nivel. Los seis grupos que se conformaron son: grupo experimental de nivel B1(EXPB1) con cinco participantes, grupo de control de nivel B1 (CTRLB1) con tres participantes, 
grupo experimental de nivel B2 (EXPB2) con cuatro participantes, grupo de control de nivel B2 (CTRLB2) con cinco participantes, grupo experimental de nivel C1 (EXPC1) con tres participantes y, finalmente, grupo de control de nivel $\mathrm{C} 1$ con tres participantes (CTRLC1).

\subsection{INSTRUMENTOS Y RECOGIDA DE DATOS}

Para medir el efecto de la instrucción explícita en la comprensión de implicaturas de los grupos de aprendientes de ELE, se diseñaron dos cuestionarios aplicados en modalidad de pretest y postest. El objetivo de esta modalidad consiste en medir la capacidad de los participantes en dos instancias, tanto de los grupos experimentales como de los grupos control: antes de participar de la sesión de instrucción explícita y al finalizar esta. En ambos casos se utilizó un cuestionario que consistió en una traducción de los items evaluativos usados por Bouton (1994) y que recoge las categorias inferenciales de Bouton (1994) y de Gallardo - Paúls (2005). Esta última autora distingue entre implicaturas generalizadas que no transgreden las máximas conversacionales de Grice e implicaturas anómalas, que sí las transgreden y que, por lo mismo, presentan mayor dificultad para su interpretación. Entre ellas están las ironías, que incluimos en este estudio.

Se trata de un diálogo que contiene una implicatura que los participantes debian interpretar con suficiente contexto para facilitarlo. La traducción de los items fue realizada contextualizando los enunciados de acuerdo al medio local y a la lengua meta. Al responder, los participantes debían elegir una de cuatro interpretaciones posibles. Se optó por presentar los ítems sin un soporte audiovisual con la finalidad de anular la variable fonética (suprasegmental) o cualquier elemento paralingüístico, a fin de acotar el estudio.

Como una forma de validar este test y poder contrastar las respuestas de los aprendientes con las de hablantes nativos, se realizó una encuesta en línea de la cual se recibieron las respuestas de 71 informantes hablantes nativos de español: 50 mujeres y 21 hombres, con una edad promedio de 30,3 años. Cada pregunta tenía solamente una respuesta correcta y dicha respuesta debía obtener la mayoría de las preferencias para que la pregunta fuera considerada válida.

\subsection{Tratamiento}

El tratamiento aplicado a los grupos experimentales consistió en una sesión de instrucción explícita sobre la inferencia de implicaturas en español. Esta sesión tuvo una duración de entre 30 a 45 minutos para cada grupo de nivel de lengua y a lo largo de ésta se expuso a los participantes a situaciones que planteaban cinco tipos de implicatura: tres correspondientes a lo que Bouton (1994) denominó ironía (1), crítica indirecta a través de implicatura (2) e implicatura de relevancia (3) y dos tipos que, dentro de la teoría de Grice (1975) se entienden como implicatura conversacional generalizada (4) 
e implicatura de cantidad (5). La elección de estos tipos de implicaturas en este trabajo responde a la iniciativa de estudiar lo que sucede con implicaturas contextualizadas o anómalas (Gallardo - Paúls, 2005) versus convencionales, ya que, según Bouton, los contextos específicos generan más problemas para la interpretación, mientras que con las implicaturas convencionales sucede lo contrario.

(1) (Debido a una mala ejecución de piano). A: ¿Te gustó cómo toqué "Claro de luna"? B: "Pensé que tocabas "La Bamba"

(2) (Sobre el informe de un estudiante). Profesor A: “¿Qué opinas del informe de Mario?" Profesor B: "Bueno... está bien escrito"

(3) (Francisco quiere saber la hora). F: “¿Qué hora es, Claudia” C: "Recién pasó el camión de la basura”

(4) (Un profesor se encuentra con un ex estudiante después de mucho tiempo). P: “¿Cómo has estado? ¿Hiciste ese viaje que tanto querias? E: Si, es decir, hice un viaje.

(5) (Una madre responde a la insistente pregunta de su hijo, sobre cuándo va a comer). $M$ : “Cuando esta señora que está aqui termine de hacer lo que está haciendo, se ponga de pie, camine hacia la cocina, saque los ingredientes, cocine el almuerzo, lo ponga en platos, y lleve los platos a la mesa".

Se incluyeron diálogos que presentaban cada uno de los tipos de implicaturas incluidos en el estudio, es decir, un total de cinco interacciones. Al terminar de leer cada segmento los participantes respondieron una pregunta formulada de la misma manera en la que se expusieron las preguntas en los instrumentos de evaluación. Luego de responder, los participantes pudieron dar su opinión respecto de sus inferencias y el instructor indicó cuál era la respuesta correcta y el porqué de la selección. Las sesiones fueron llevadas a cabo completamente en español.

\section{Resultados}

La información recabada a través de los instrumentos descritos fue expresada en un porcentaje de respuestas correctas obtenidas por cada uno de los 6 grupos en ambas evaluaciones. El porcentaje del pretest se comparó con los porcentajes del postest (ver Gráfico 1) y se aplicó pruebas estadísticas para establecer si había diferencias significativas entre e intra grupos. 


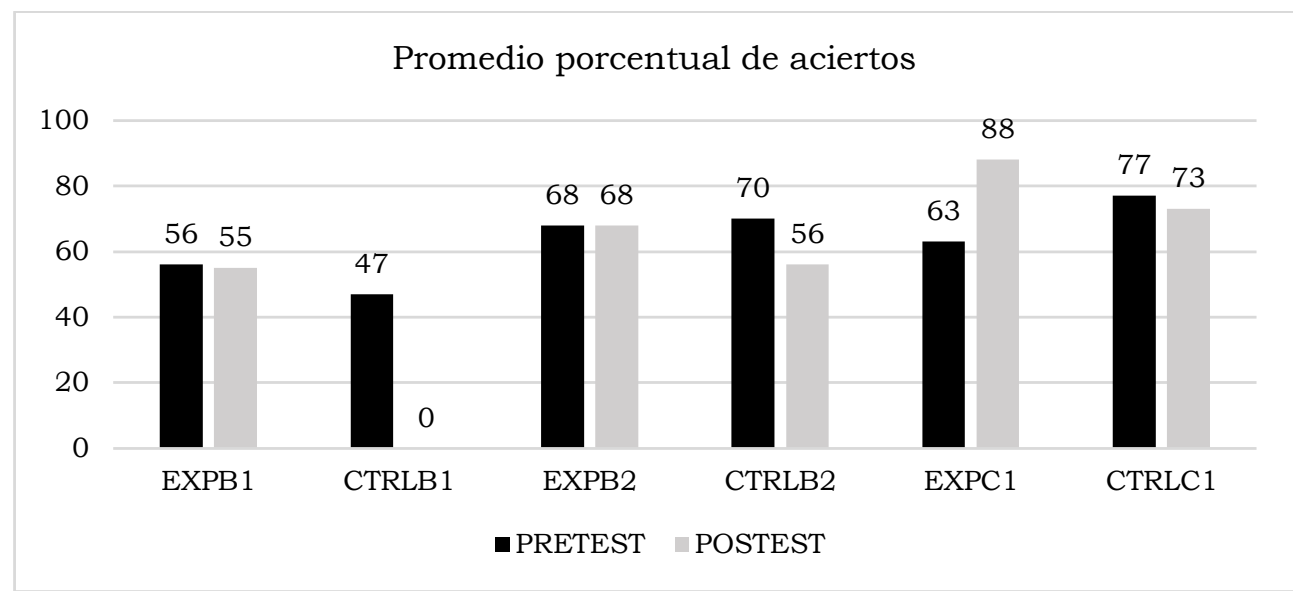

Gráfico 1: Comparación de promedios de aciertos por grupo en el pretest y en el postest

En el gráfico 2 se comparan los porcentajes de aciertos según tipo de implicatura obtenidos por todos los participantes en ambas evaluaciones.

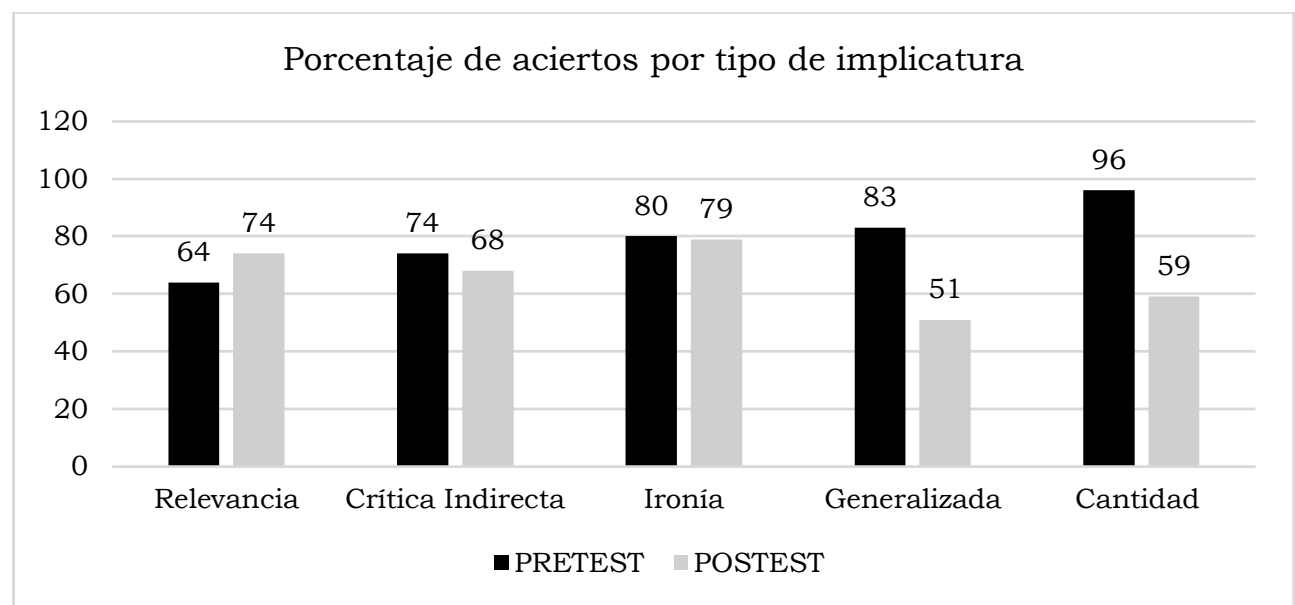

Gráfico 2: Comparación de porcentaje de aciertos por tipo de implicatura en pretest y postest

Una vez obtenidos los resultados expuestos en los gráficos anteriores, se planificó una serie de análisis estadísticos para establecer si las diferencias entre los resultados obtenidos por los distintos grupos en ambas evaluaciones eran estadísticamente significativos. Se consideró innecesario aplicar dichos análisis en los siguientes casos:

- Grupo EXPB1: que solamente registró una disminución de 1\% en el promedio de acierto entre las muestras.

- Grupo CTRLB1: ya que los participantes no estuvieron disponibles para la aplicación del postest. 
- Grupo EXPB2: puesto que los promedios de aciertos son idénticos.

- Grupo CTRLC1: dado que solamente se registra una disminución de 4\% en el promedio de acierto entre las muestras.

En el caso del grupo CTRLB2 se consideró útil aplicar una prueba t para medias de dos muestras emparejadas y así determinar si la diferencia entre los promedios obtenidos por el grupo en ambas evaluaciones era estadísticamente significativa. A continuación se exponen los resultados de esta prueba:

\begin{tabular}{|lll|}
\hline Prueba t para medias de dos muestras emparejadas & 0.7 \\
\hline Media & 0.8 & 0.575 \\
Varianza & 0.925 & 0.029166667 \\
Observaciones & 0.009166667 & 4 \\
Coeficiente de correlación de & 4 & \\
Pearson & & \\
Diferencia hipotética de las medias & -0.560611911 & \\
Grados de libertad & 0 & \\
Estadistico t & 3 & \\
P(T<=t) una cola & 2.940588176 & \\
Valor critico de t (una cola) & 0.030240751 & \\
P(T<=t) dos colas & 2.353363435 & \\
Valor critico de t (dos colas) & 0.060481503 & \\
\hline
\end{tabular}

Tabla 1: Prueba t Student para los resultados del grupo CTRLB2 en el pretest y el postest

La prueba estadística de $\mathrm{t}$ Student permite evaluar si los resultados del grupo CTRLB2 en el pretest y en el postest difieren entre sí de manera significativa respecto a sus medias. Como se puede apreciar en la Tabla 1, el valor $\mathrm{t}$ de 2,94 con 3 grados de libertad y un nivel de significancia de 0,05 es superior al valor critico de $\mathrm{t}$ de 2,35 , lo cual permite afirmar que la diferencia en los resultados obtenidos por el grupo en ambas evaluaciones es estadisticamente significativa.

También se utilizó una prueba t Student para determinar si la diferencia en los promedios obtenidos por el grupo EXPC1 en el pretest y en el postest era significativa. Es decir, si el aumento de $25 \%$ en el postest podía ser atribuido a la sesión de instrucción explícita. No obstante, como se puede apreciar en la tabla 2 con 3 grados de libertad y un valor crítico de t de 2,35, el valor t de 1,1 no permite afirmar que la diferencia entre los promedios obtenidos por el grupo EXPC1 en el pretest y el postest sea estadísticamente significativa. Por lo mismo no es posible afirmar que el aumento de $25 \%$ entre los resultados de ambas evaluaciones se deba a la sesión de instrucción explícita y no al azar.

\begin{tabular}{|lll|}
\hline \multicolumn{2}{|l|}{ Prueba t para medias de dos muestras emparejadas } & \\
\hline & 0.66 & 0.75 \\
\hline Media & 0.9575 & 0.91 \\
Varianza & 0.007225 & 0.0036 \\
Observaciones & 4 & 4 \\
Coeficiente de correlación de & & \\
Pearson & 0.333333333 &
\end{tabular}


\begin{tabular}{|ll|} 
Diferencia hipotética de las medias & 0 \\
Grados de libertad & 3 \\
Estadistico t & 1.102491821 \\
$\mathrm{P}(\mathrm{T}<=\mathrm{t})$ una cola & 0.175377137 \\
Valor critico de t (una cola) & 2.353363435 \\
$\mathrm{P}(\mathrm{T}<=\mathrm{t})$ dos colas & 0.350754273 \\
Valor critico de t (dos colas) & 3.182446305 \\
\hline Tabla 2: Prueba t de Student para los resultados del grupo EXPC1 en el pretest y el postest
\end{tabular}

\section{Discusión DE RESUlTADOS Y CONCLUSIONES}

Los resultados obtenidos en el estudio de Bouton (1994) indican que, aquello que hace que un tipo de implicatura sea más dificil de interpretar por hablantes no nativos es el tipo de razonamiento necesario para descifrar su significado contenido. En otras palabras, las implicaturas convencionales son más fáciles de interpretar que aquellas que no lo son. Efectivamente, en los resultados del pretest se puede apreciar que las implicaturas que muestran mayor dificultad son del tipo relevancia. Sin embargo, en los resultados obtenidos en el postest se observa que las implicaturas del tipo ironía y relevancia parecen ser interpretadas con mayor facilidad. Esto implica que la sesión de instrucción tuvo un efecto positivo en la interpretación de aquellas implicaturas que en un comienzo les resultaban más complejas a los aprendientes. El porcentaje de aciertos en las preguntas que contienen implicaturas del tipo relevancia aumentó un 10\%; el de crítica indirecta descendió un $6 \%$ y el de ironía se mantuvo casi inmutable, descendiendo solo un $1 \%$. Finalmente las implicaturas de tipo generalizada y de cantidad descendieron un $32 \%$ y un $37 \%$ respectivamente.

Con respecto al primer objetivo de investigación: determinar si existe alguna relación entre la mayor competencia gramatical de los aprendientes y la comprensión de implicaturas, se puede afirmar que por lo menos en los tres niveles de competencia lingüística estudiados no fue posible establecer que la diferencia de desempeño fuera estadísticamente significativa. Por lo tanto, si bien los resultados preliminares muestran una relación entre el nivel de competencia y la comprensión de implicaturas por parte de los aprendientes de ESL, no es posible afirmarlo categóricamente. La comparación que ofrecen Cignetti y Di Giuseppe (2015) sobre los trabajos de Bouton (1994) y Kubota (1995) permite entender que el nivel de competencia en la lengua objeto influye en la comprensión de implicaturas por parte de los aprendientes. Es decir, la comprensión de implicaturas por parte de hablantes no nativos se ve beneficiada cuando la competencia lingüística de los aprendientes es lo suficientemente alta para permitirles exponerse a situaciones donde deban calcular implicaturas en la lengua objeto. Una réplica del presente estudio, con acceso a un mayor número de participantes, podría entregar la confirmación estadística que avale las posturas de Bouton y Kubota.

Al respecto, descartando los resultados de las pruebas estadísticas, en el único grupo que se pudo apreciar un cambio positivo después de la sesión de instrucción explícita es el grupo EXPC1 que aumentó su promedio de 
aciertos desde un $63 \%$ en el pretest a un $88 \%$ en el postest. A pesar de que esta diferencia no es estadísticamente significativa, dicho incremento podría indicar que con mayor cantidad de sesiones es posible que la instrucción explicita sobre implicaturas tenga un efecto positivo en su interpretación por parte de aprendientes de ESL de nivel $\mathrm{C} 1$, no asi en niveles que aun necesitarian mayor desarrollo en su competencia lingüística. Lo anterior contrasta con la idea de que el desarrollo pragmático precede al gramatical cuando se aprende una L2, según señala Bialystok (1993).

Para el segundo objetivo de esta investigación: determinar si existe algún tipo de implicatura conversacional que sea más difícil de comprender por los aprendientes, se puede señalar que el comportamiento de los participantes con respecto a la interpretación de distintos tipos de implicaturas fue variable y no indica dificultades significativas de ningún tipo sobre otro, si bien en el pretest se apreció mayores problemas con las implicaturas de relevancia, tal como lo había advertido Bouton (1994). Un estudio que atienda a esta dificultad con acceso a un mayor número de participantes podría confirmar los resultados que arroja esta investigación.

En cuanto al tercer objetivo de investigación: determinar el efecto que tiene una sesión de instrucción explícita sobre la comprensión de implicaturas conversacionales en español por parte de aprendientes de ESL, cabe señalar que el tratamiento pareciera no tener efecto en los aprendientes de los dos niveles de menor competencia gramatical (B1 y B2), si bien esto no se puede asegurar con respaldo estadístico. Por otra parte, el grupo del nivel más alto (C1) produjo un aumento importante de $25 \%$ de aciertos en el instrumento aplicado, lo cual podría indicar que los niveles más altos de competencia lingüistica en una SL se benefician más del efecto de las sesiones de instrucción explícita en su capacidad para inferir implicaturas en español.

A continuación se propone un plan potencial de acción para terminar de resolver las interrogantes que quedan pendientes y solucionar los problemas logísticos de los instrumentos evaluativos que se utilizaron en este estudio. Como resultado de los puntos expuestos en la discusión de los párrafos anteriores se estima que una nueva intervención debería tomar en cuenta lo siguiente:

Considerar solamente dos tipos de implicaturas, las del tipo relevancia que según Bouton (1994) son poco convencionales y las de tipo generalizadas que son más formulaicas y dependen en mayor grado de las expresiones utilizadas para su interpretación.

Investigar solo con participantes de nivel $\mathrm{C} 1$, ya que este nivel parece haber respondido a la sesión de instrucción explícita.

Resguardar la fiabilidad de los cuestionarios mediante el cálculo de consistencia interna (coeficiente Alpha de Cronbach). De este modo se podría estar seguro de que nos encontramos con instrumentos con garantías suficientes de fiabilidad. Finalmente, y con el objetivo de analizar la validez del constructo del cuestionario, se realizaria un análisis factorial de componentes principales. 
Realizar más de una sesión de instrucción explícita de media hora y disenar cinco actividades referidas a implicaturas para que sean aplicadas a los aprendientes por la misma persona encargada de hacerles clases de ESL.

Si se puede concluir con apoyo estadístico que la instrucción explícita sobre implicaturas ayuda a los aprendientes a interpretarlas mejor, se puede tener otra razón para incluir temas de desarrollo de competencia pragmática en el currículo de ESL. En caso contrario, la idea de utilizar la instrucción explícita como medio de concienciación pragmática en el aula de ESL podría ser descartada, para centrarse más en el estudio del efecto de otros tipos de actividades con un enfoque más inductivo o de naturaleza comunicativa.

\section{REFERENCIAS}

BARDOVI-HARLIG, K. (1996): "Pragmatics in Language Teaching", Bringing Pragmatics and Pedagogy Together. Pragmatic and Language Learning. Monograph Series. 7(1), pp. 21-39.

BARDOVI-HARLIG, K. \& DÖRNYEI, $Z$. (1998): "Do language learners recognize pragmatic violations? Pragmatic versus grammatical awareness in instructed L2 learning”. TESOL Quarterly, 32, $233-262$.

BOUTON, L. (1994): "Can NNS Skill in Interpreting Implicature in American English Be Improved through Explicit Instruction? A Pilot Study", Pragmatic and Language Learning. Monograph Series. 5(1), pp. 89-109.

CIGNETTI, L. M., \& DI GIUSEPPE, M. S. (2015). "Pragmatic awareness of conversational implicatures and the usefulness of explicit instruction", Revista Nebrija de Lingüistica Aplicada, 9(0). Publicación electrónica: https://www.nebrija.com/revista-linguistica/files/articulosPDF/articulo_56598f3498e3f.pdf (Fecha de consulta $3 / 9 / 17)$.

CLENNELL, C. (1999): "Promoting Pragmatic Awareness and Spoken Discourse Skills with EAP Classes", ELT Journal, 53(2), pp. 83-91.
CONSEJO DE EUROPA (2002): Marco Común Europeo de Referencia para la enseñanza de lenguas. Madrid: Ministerio de Educación, Cultura y Deporte, Subdirección General de Cooperación Internacional, para la edición impresa en español.

COOK, M. \& LIDDICOAT, A. (2002): "The development of comprehension in interlanguage pragmatics: The case of request strategies in English", Australian Review of Applied Linguistics, 25: $19-39$.

COX, J. (2015): “Explicit Instruction, Bilingualism and the Older Adult Learner", Studies in Second Language Acquisition, 2015, pp. 1-30.

ESLAMI-RASEKH, Z. (2004): "Raising the Pragmatic Awareness of Language Learners", ELT Journal, 59(3), pp. 199-208.

FORDYCE, K. (2013): “The Differential Effects of Explicit and Implicit Instruction on EFL Learners' Use of Epistemic Stance", Applied Linguistics, 35(1), pp. 628.

GALLARDO-PAÚLS, B. (2005): "Categorias inferenciales en pragmática clinica", Revista de neurología, 41(1), pp. 65-71.

GAZDAR, G. (1979): Pragmatics: Implicature, Presupposition and Logical Form. New York: Academic Press. 
GRICE, H. P. (1975): "Logic and Conversation", P. Cole y J. Morgan (eds.): Syntax and Semantics, 3: Speech Acts, New York: Academic Press, pp. $41-58$.

GRICE, H. P. (1989): Studies in the Way of Words. (3a. Ed.). Cambridge, Estados Unidos: Harvard University Press.

KASPER, G., ROSE, K. (2002): Pragmatics in Language Teaching. (1a. Ed.). Cambridge, Estados Unidos: Cambridge University Press.

KASPER, G., \& SCHMIDT, R. (1996): "Developmental Issues in Interlanguage Pragmatics", G. Kasper y R. Schmidt (Eds.)

KOIKE, A. (1989). "Pragmatic Competence and adult Second language Acquisition: Speech Acts in Interlanguage", The Modern Language Journal, vol. $73, \mathrm{~N}^{\circ} 3$, 279 - 289. National Federation of Modern Language Teachers Associations.Studies in Second Language Acquisition, 18, pp. 1 49-169.

KUBOTA, M. (1995): "Teachability of Conversational Implicature to Japanese EFL learners", IRLT (Institute for Research in Language Teaching) Bulletin, 9 (1), pp. 35-67.

MARTÍNEZ-FLOR, A. \& ALCÓN, E. (2004): "Developing Pragmatic Awareness of Suggestions in the EFL Classroom: A Focus on Instructional Effects", Canadian Journal of Applied Linguistics (CJAL), 10 (1), pp. $47-76$.

MCMANUS, K. (2017): "L1 Explicit Instruction Can Improve L2 Online and Offline Perfor- mance", Studies in Second Language Acquisition, 39 (3), pp. 459 - 492.

MEDGYES, P. (1992): "Native or native: Who's worth more?", ELT Journal, 46 (4), pp. $340-349$.

NIEZGODA, K., \& RÖVER, C. (2001). "Pragmatic and grammatical awareness". In K. R. Rose \& G. Kasper (Eds.), Pragmatics in language teaching (pp. 63-79). Cambridge: Cambridge University Press.

PEARSON, L. (2006): "Patterns of Development in Spanish L2 Pragmatic Acquisition: An Analysis of Novice Learner's Production of Directives", The Modern Language Journal, 90 (4), pp. 473495.

ROSE, K. (1994): "Pragmatic Consciousness-Raising in an EFL context", Pragmatics and Language Learning. Monograph Series. 5 (1), pp. 52-63.

SCHMIDT, R. (1990): “The Role of Consciousness in Second Language Learning", Applied Linguistics, 11 (2), pp. $129-158$.

SCHMIDT, R. (1993a): "Consciousness, learning, and interlanguage pragmatics", G. Kasper \& S. Blum-Kulka (Eds.), Interlanguage pragmatics, pp. 21-42. New York: Oxford University Press.

SCHMIDT, R. W. (1993b): "Awareness in second language acquisition", Annual Review of Applied Linguistics, 13, pp. 206-226.

WIDDOWSON, H. (1992): "ELT and EL teachers: Matters arising”, ELT Journal, 46 (4), pp. 333 - 339. 